\title{
Research on VaR Computing System of Commercial Banks Based on Accurate Scenario Data
}

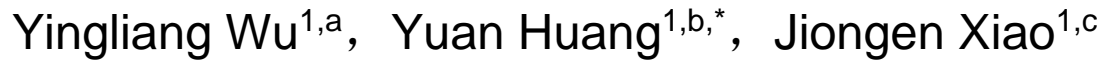 \\ ${ }^{1}$ School of Economics and Commerce, South China University of Technology, Guangzhou, \\ Guangdong, China
}

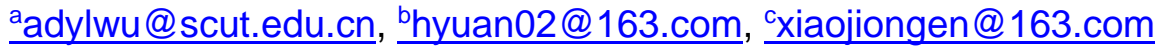

Keywords: Scenario Calculation, VaR calculation, System Architecture, Data Accuracy.

\begin{abstract}
Basing on scene analysis and VaR calculation, this paper discussed system architecture of scenario calculation and accurate data's importance of system model from the view of system theory. We also discussed several kinds of data errors and their processing methods to avoid the error of data. Then we discussed scenario engine architecture and detailed described function of each module as well as the process of data extraction to the scenario analysis. Last, we discussed the construction of VaR system and carried out a case study basing on stock price of Google in later two years. Through the analysis of case, we verified the feasibility of the system architecture.
\end{abstract}

\section{商业银行基于准确的情景数据VaR计算系统研究}

\author{
吴应良 ${ }^{1, a}$ ，黄媛 $1, b,{ }^{\star}$ ，肖畑恩 ${ }^{1, c}$ \\ 1华南理工大学经济与贸易学院, 广州, 广东, 中国

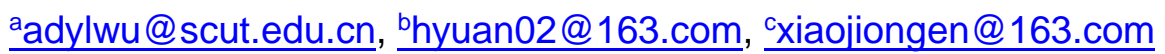

关键词: 情景计算, VaR计算, 系统架构, 数据准确性

中文摘要. 本文以情景计算为主题, 重点从 $\mathrm{VaR}$ 的计算出发, 讨论了情景计算系统的架构, 分 析准确数据对于系统模型的重要性, 讨论了数据错误的几种情况以及处理方法, 从而避免了 数据错误。以系统论的观点, 对情景分析和VaR计算系统进行论述。情景引擎架构的讨论, 从 总体架构出发, 再详细论述每个模块功能。对系统流程进行了描述, 详细描述了数据的提取 到情景分析结果的生成的整体流程。对 $\mathrm{VaR}$ 系统的构建进行了论述, 并且以谷歌最近两年的 股票价格数据为基础, 进行实例分析。通过实例的分析, 验证了系统架构的可行性。

\section{1. 引言}

情景分析（Scenario Analysis，SA）,基于银行内，外实际历史数据的情景分析，在运用 内部数据的基础上, 增加外部数据, 以确保数据的准确性, 从而确保计算结果的准确性 ${ }^{[1]}$ 。情 景分析主要分成两个方面, 一方面是对每日的情景进行研究分析, 另一方面是根据银行内外 部因素的重大变化提出各种关键假设, 进行详细、严密的推理, 计算可能的变化结果, 最终 为决策服务。情景分析和压力测试 (Stress Testing) 有很多相似之处, 都是从各种内外部因素 的重大变化进行分析。理论界对于情景分析和压力测试的范畴界定目前仍然有争论, 但是在 实践中情景分析和压力经常是同时进行, 其中负面的测试就是压力测试 [2]。Brawn 和 Cathcart （2006）认为, 压力测试的方法包括敏感性分析和情景分析等, 敏感性分析是指在保持其他 
条件不变的前提下，研究单个或者少数几个的重要风险因素的变化对金融机构可能产生的影 响，情景分析则是假设分析多个因素的变化产生的影响 ${ }^{[3]}$ 。

《巴塞尔协议 II 》是为银行设定最低的风险资本要求，VaR（Value at Risk）就是衡量银 行风险的工具之一，也是情景分析的工具之一。JP摩根是最先披露其 $V a R$ 的银行之一。VaR 在市场正常波动下，某一金融资产或证券组合的最大可能损失。更为确切的是指，在一定概 率水平 (置信度) 下，某一金融资产或证券组合价值在未来特定时期内的最大可能损失。外 汇利率、国内利率、金融衍生品、股票等计算 VaR会使用不同的模型。VaR计算的分析和研 究, 不应当仅仅局限于模型的建设, 或者是模型的改进, 更应当从系统的角度, 整体的视觉 对 $\mathrm{VaR}$ 计算系统进行论述。

\section{VaR的计算}

在确保数据的准确性的基础上，开始运用 VaR 这一工具来讨论情景分析。VaR 假设目前 的投资组合在一段时间内冻结，将目前的投资组合的头寸和投资组合到期前的不确定风险因 素均考虑在内。VaR 是在目标区域内发生的最大损失的最小可能性概率时间, 这涉及到两个 定量因素: 目标区域和置信水平。

假设 $\mathrm{C}$ 为置信水平, $\mathrm{L}$ 为损失, 用正数表示, $\mathrm{VaR}$ 也是正数。 $\mathrm{VaR}$ 一般定义为最小损失、 绝对值, 如下:

$$
P(L>V A R) \leq 1-C
$$

例如,假设 $99 \%$ 置信水平内, 或者 $\mathrm{C}=0.99, \mathrm{VaR}$ 是临界损失, 即发生更大损失的概率小于 $1 \%$ 。

$\mathrm{VaR}$ 的计算包括德尔塔-正态分布法，历史模拟法，蒙特卡罗模拟法。这三种方法各有 优点和缺点。在实践中也都有使用，但是本文讨论的情景分析法主要是基于历史模拟法。

历史模拟法是金融商品之风险因子计算过去一段时间内的资产组合风险收益的频率分 布,通过找到历史资料求出其报酬率，然后搭配目前持有资产的投资组合部位，则可以重新 建构资产价值的历史损益分配, 然后对资料期间之每一交易日重复分析步骤, 如果历史变化 重复时，则可以重新建构资产组合未来报酬的损益分配。

假设现在的时间为 $\mathrm{t}=0^{\prime} \mathrm{Si}(\mathrm{t})$ 为第 I项资产在时间 $\mathrm{t}$ 的价格,以历史模拟法来估算未来一天的 风险值的程序:

（1）选取过去 $N+1$ 天第I项资产的价格作为模拟资料; 例如首先找出过去一段时间（假设 是201天）的股票收盘价： $\operatorname{Si}(-1) 、 \operatorname{Si}(-2) \ldots \operatorname{Si}(-200) 、 \operatorname{Si}(-201)$ 。

(2) 将过去彼此相邻的 $N+1$ 笔价格相减, 就可以求得 $\mathrm{N}$ 笔该资产每日的价格损益变化 量; 例如: $\Delta 1=\mathrm{Si}(-1)-\mathrm{Si}(-2) 、 \Delta 2=\mathrm{Si}(-2)-\mathrm{Si}(-3) 、 \Delta 200=\mathrm{Si}(-200)-\mathrm{Si}(-201)$ 。

（3）步骤 2 代表的是第项资产在未来一天损益的可能情况（共有 $\mathrm{N}$ 种可能情形）, 将变 化量转换成报酬率, 就可以算出 $\mathrm{N}$ 种的可能报酬率，如下所示。

$$
\begin{aligned}
& R_{1}=\frac{s_{i}(-1)-S_{i}(-2)}{S_{i}(-2)} \\
& R_{2}=\frac{S_{i}(-2)-S_{i}(-3)}{S_{i}(-3)} \\
& \cdots \cdots \cdots \\
& R_{N}=\frac{s_{i}(-\mathrm{N})-S_{i}(-\mathrm{N}-1)}{S_{i}(-\mathrm{N}-1)}
\end{aligned}
$$

(4) 将步骤3的报酬率由小到大依序排列, 并依照不同的置信区间找出相对应分位数的 临界报酬率。

(5) 将目前的资产价格 $\operatorname{Si}(0)$ 乘以步骤4的临界报酬率，得到的金额就是使用历史模拟法 所估计得到的风险值。

由以上的计算过程可以知道资产价格是相对固定的, 反映情景变化的是价格损益变化量 或者报酬率, 因此在情景分析和压力测试的时候, 主要是对损益变化量或者报酬率的假设。 
构建系统的时候主要从两个方面考虑，一是要确保数据的准确性，二是要满足根据用户的需 要进行各种动态情景数据的输入。

\section{3. 系统的设计}

为了适用情景分析的需求和 VaR 计算所需数据的输入及展现。需要从系统的架构进行讨 论, 接着讨论不同的情景分析过程中的数据整理, 实现系统的数据自动化处理, 最后讨论系 统如何生成数据。

\section{1 情景计算系统总体架构}

系统的架构图主要包含了数据的输入和结果的展现, 由于情景数据的动态需求, 因此系 统的输入部分必须包含两部分既可以动态输入也可以系统自动获取。其总体架构如图1所示:

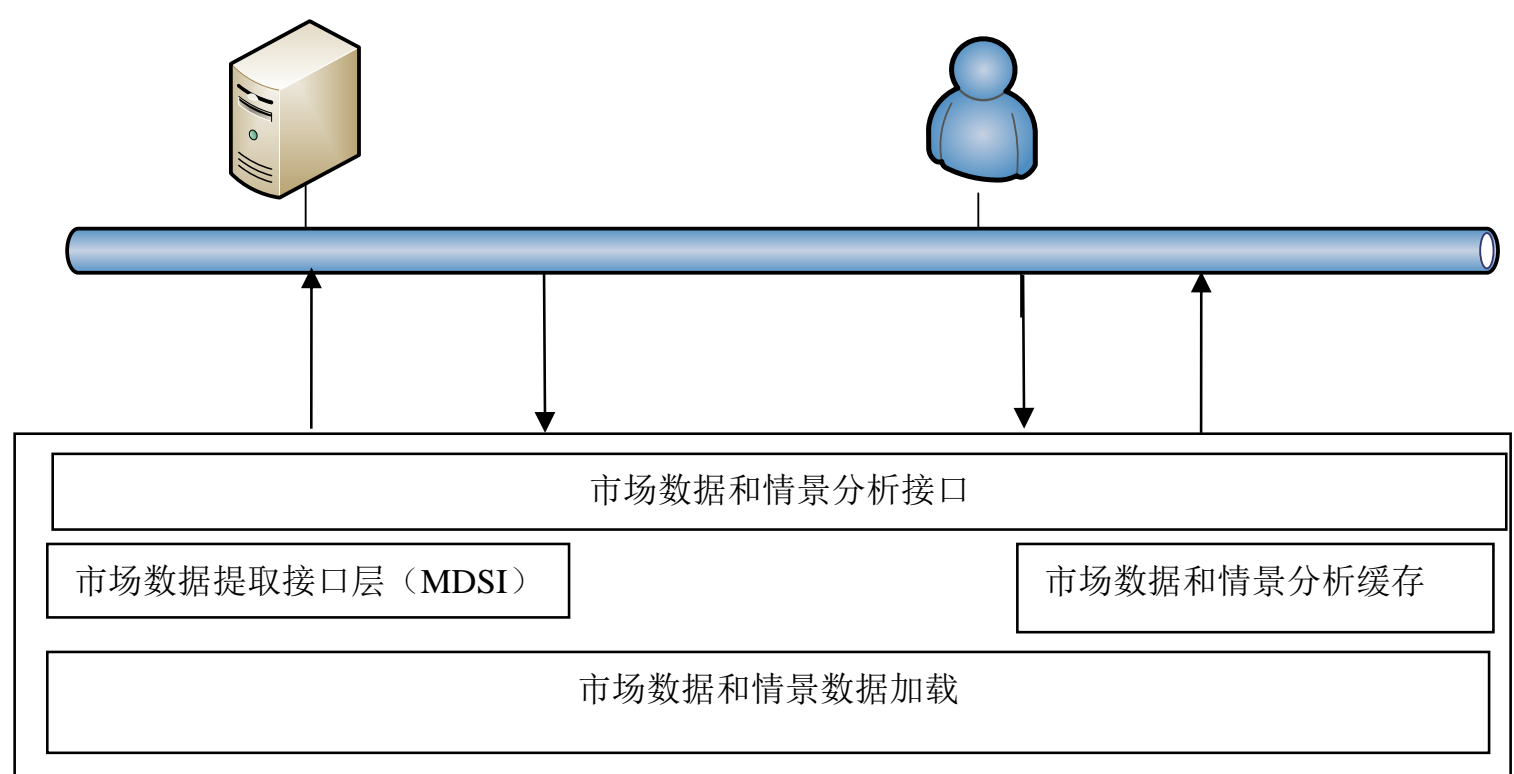

压力和风险价值情景计算引擎（SVSE）

市场数据提取（MDE）

Oracle

图1 情景计算系统总体架构

系统架构图1中各个模块的具体功能如下:

(1) Oracle数据库: 主要负责市场数据的获取, 标准化, 转换和整合后, 进行存储。

(2) 市场数据提取（MDE）：主要负责静态数据和时间序列数据的提取。

（3）压力和风险价值的情景引擎（SVSE）：是基础的压力测试和风险价值参数的情景 进行计算。

（4）市场数据和情景分析接口（MDSI）: 市场数据的接口主要是接收各种特殊的情景 数据的输入，同时通过MDSI把情景计算结果发送到VAR计算系统中。

多情景计算系统详细架构, 主要考虑系统的弹性, 历史数据的准确性, 情景数据的动态 性，计算结果的准确性，计算过程的高效性，其架构如下图2所示： 


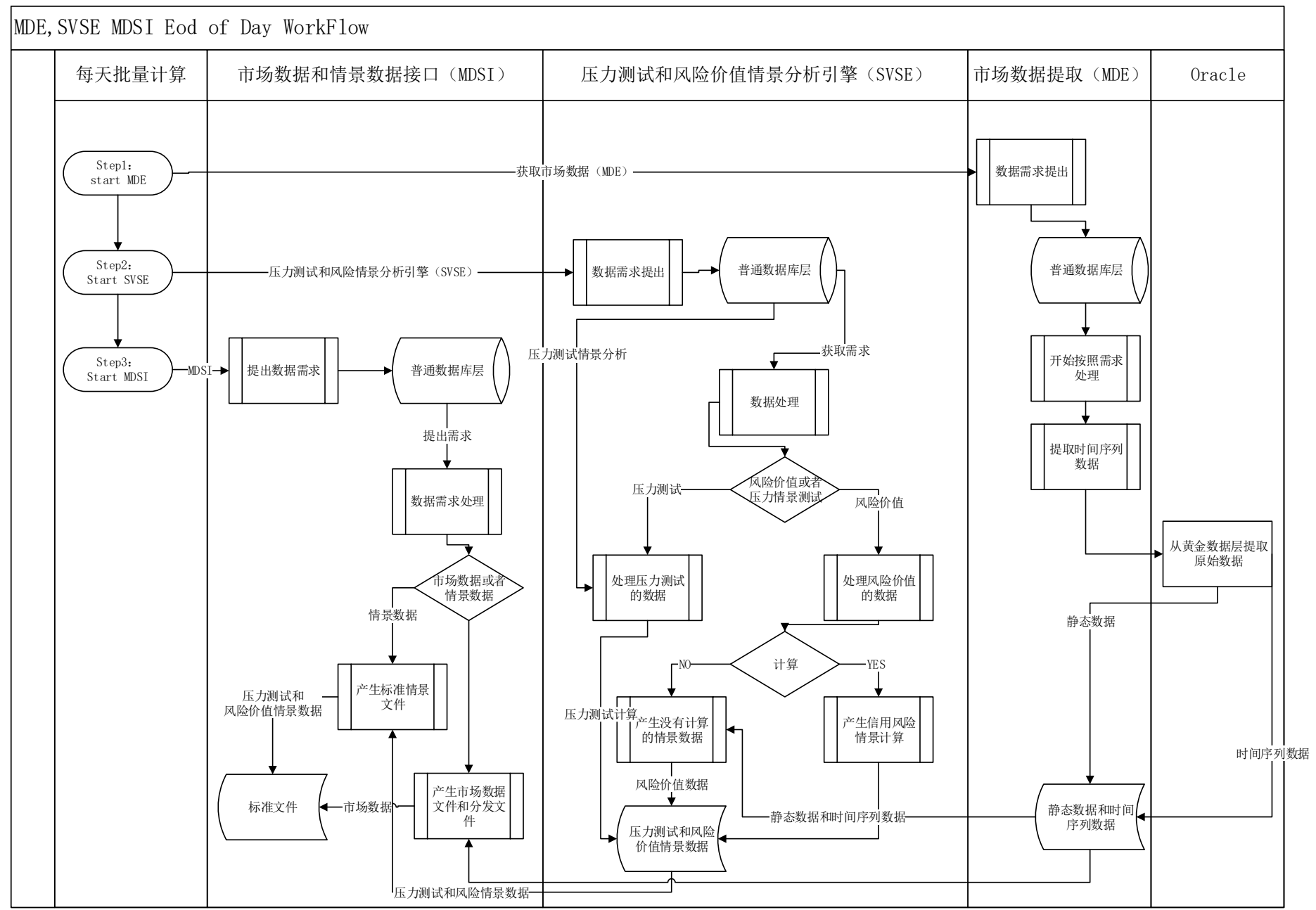

图2 多情景计算系统架构设计

(1) 普通模块层

普通模块层用于连接其他数据, 提供以下服务: 数据接入: 数据库, 服务器上的文件, 或者是NAS上的文件安全和授权的工作, 记录程序的 $\log$ 文件, 文件格式的转换和处理, 提 供Oracle数据的存储接口

(2) 市场数据的获取

市场数据的获取简称 (MDE) 为情景分析数据的产生和分发提供单一的接口, Oracle存 储着市场数据, 进行数据清洗, 整理后生成的黄金数据。系统平台满足的需求是提供市场数 据和情景数据结果, 包括 $\mathrm{VaR}$ 的计算结果。每天对数据进行计算并生成结果。从Oracle读取 市场数据并且写到文件中或者NAS中，这有助于运用GRID引擎进行计算多维度的情景，并 提供分析的结果。情景分析引擎将会整合大量内容和外部的计算工具, 同时借助于grid来获 得最终的计算结果。并把结果写到文件服务器或者NAS中。由于现今对于大数据的读写, 主 要都是通过非关系型数据库进行的, 因此未来可以选择HBase或者Cassandra来存储数据, 并 加快读写速度。稳定而成熟的技术则采用文件系统, 并利用Oracle建立索引, 以提高读写速 度。

（3）压力测试和风险价值情景计算引擎（SVSE）

情景计算引擎是系统的核心, 对于情景分析结果的产生和风险价值计算都非常重要。它 首先会产生计算的情景数据 (经过计算的主要是通过第三方计算软件进行平滑化的计算) 和 非计算情景数据（是指直接读取Oracle处理整合后的数据）。每天计算的内容包括业务结束 日的情景计算和每日的数据处理。另外还承担着突发情况的计算, 例如某地方突然地震, 计 算该事件对该国股票的影响和对世界经济的影响。 


\section{（4）市场数据和情景分析接口（MDSI）}

MDSI使用来转换和分发的工具, 它的功能是提供用户上传数据或者获取数据的接口, 具体包括以下四项: 提出数据需求, 数据转换, 数据分发, 更新或者上传数据到情景计算系 统中。

（5）全球用户接口（Desktop UI）

用户可以通过这个平台获取数据或者通过这个平台直接在系统中进行一些数据的提取。 提取临时需要的数据，也可以通过这个平台获取历史上的情景计算结果。

\section{2 数据准确处理}

从情景计算架构图中可以看出风险模型依赖于金融时间序列数据以及交易主体的静态数 据的准确性, 经过处理后的数据存储于Oracle 数据库中。数据分类主要包括静态数据和时 间序列的动态数据, 静态数据主要是指交易主体的各种属性, 包括企业的名称, 编号, 类 型, 地域, 行业等。时间序列动态数据主要是指随着时间变化的数据, 例如每天某一时刻的 股价, 利率等, 它是时刻变化的。每天交易规模巨大, 产生数以GB计的数据, 确保数据的 准确性是一个研究的重要课题。因此如何设计一个准确的数据输入系统成为重中之重。一旦 输入数据出现错误，后续的工作无论多完美，最终的结果也是错误的。

从系统架构图中, 可以知道数据来源于内部也来源于外部, 为了确保外部购买数据的准确 性，外部数据经常向多个供应商采购。知名的数据供应商包括MarkIT, Bloomberg,

Sophis, Moody's等。多个供应商提供的数据不一定准确, 或许存在错误或者遗漏。所以必 须进行一定的处理, 生成标准化的数据。只有标准化的数据才能为后续的工作所用, 才能被 有效处理, 生成最后准确的数据。主要的数据问题有以下几种:

(1) 不同数据源格式不同

不同的供应商提供了相同的数据，但是数据之间使用的格式是不同的，例如供应商 $\mathrm{A}$ 提 供的数据使用的 $\mathrm{xml}$ 格式, 运用各种元数据定义数据, 而供应商B提供的是CSV格式, 运用 “, ”来对数据进行分隔，供应商 $\mathrm{C}$ 有可能提供的数据是运用“”来分隔数据。对数据进行标准 化是非常有必要的, 只有标准化的数据才能比较其准确性。

(2) 数据定义不同

数据定义不同，指的是数据供应商对同一个事物的定义是不同的。例如供应商 $\mathrm{A}$ 提供的 公司信用评级是按照最好为 $\mathrm{AAA}, \mathrm{AA}, \mathrm{A}, \mathrm{BBB}, \mathrm{BB}$.....但是对于供应商提供的公司信用 评级使用的 $\mathrm{A}+, \mathrm{A}, \mathrm{A}-, \mathrm{B}+, \mathrm{B}, \mathrm{B}-$ 这种方式, 那么同样也必须对数据转化, 标准化。

\section{(3) 数据单位不同}

数据单位不同，指的是数据供应商之间采用的单位不同，例如提供 $\mathrm{A}$ 公司的股价使用的 是以美元为计量单位, 而另一个供应商提供的股价使用的是英镑为单位, 有可能这个两个数 据都是准确的, 但是他们由于单位的不同而数值不同, 无法进行比较, 这种情况下也必须进 行标准化。类似的还存在于其他的数值的单位定义问题。

(4) 数据准确性

数据的准确性问题，不同的数据供应商提供的数据每天都在 $1 \mathrm{G}$ 以上，如何确定其中那 些是正确的, 那些是错误的, 这必须依赖于前期的标准化, 以股价为例, 分别拿到了 A, B, $\mathrm{C}$ 三个公司的数据, 其中 $\mathrm{A}, \mathrm{B}$ 公司一样, $\mathrm{C}$ 公司是不同的, 那么说明 $\mathrm{C}$ 公司的数据是不准 确的。但是在某些情况下也不能简单使用这样的规则, 而是制定优先级。即当出现数据差异 时, 优先选择其中一家公司的数据。根据这家公司提供的历史数据准确性做判断的。当然特 别重要的数据也可以在系统设计的时候抛出异常, 或者记录下来, 最终由用户决定选择以那 个数据为准。

\section{(5) 数据缺失}

数据缺失的问题则是因为所有数据供应商都未能提供的数据。例如现在某个模型的计算 需要用到七年前 10 月 1 日的数据, 但是这个数据是缺失的, 通过获取 10 月以前的所有数据并 
且获得10月 2 日以后的部分数据, 那么这个时候, 可以用自回归滑动平均（ARMA）模型, 推算出 10 月 1 日的数据, 并且把它作为输入数据参与进一步的计算。

以上工作是为了确保输入数据的准确性, 这也保证了输出结果的准确性。风险价值是衡量 在现有经营状况下出现不利情况时, 风险敞口的指标。因此系统的设计必须考虑保证数据准 确性，数据处理部分的系统架构图3如下所示:

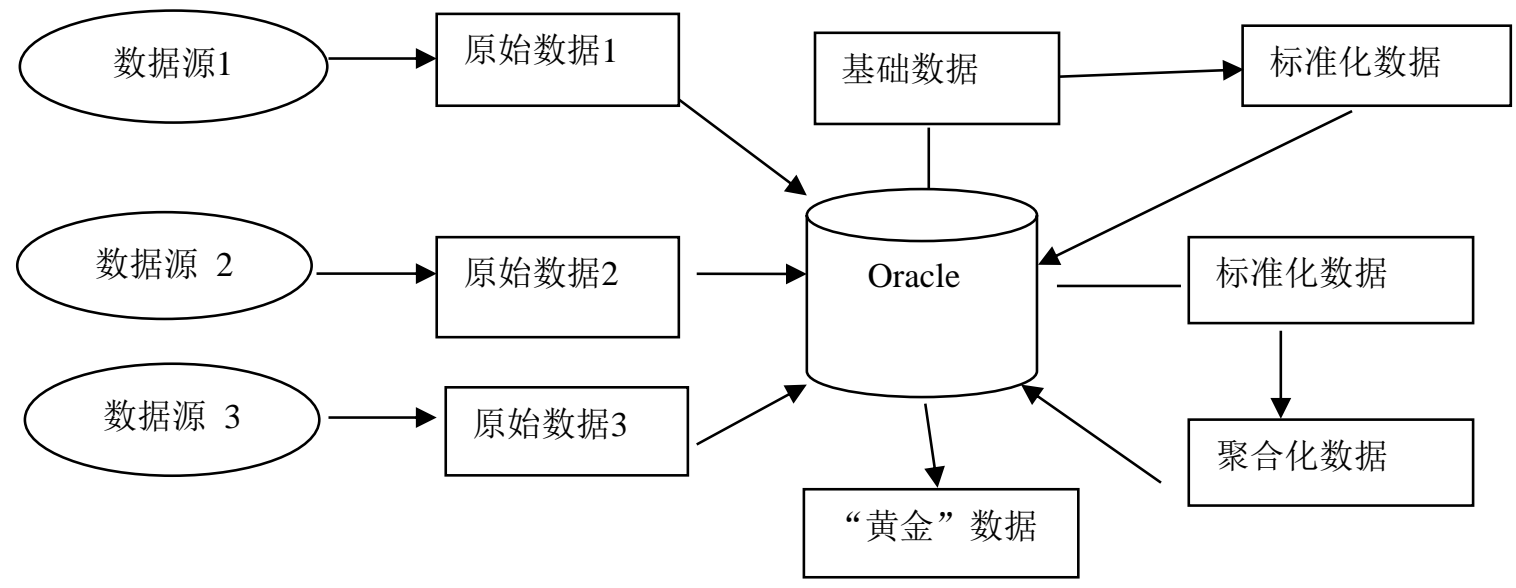

图3 数据处理架构

以上数据处理架构图主要分为三部分:

步骤1: 把数据标准化, 去除格式的差别; 步骤 2 : 标准化数据, 进行对比聚合, 对静态 数据进行比较和检验, 生成静态数据的最终数据; 步骤 3 : 再对时间序列数据进行计算, 检 验其中数据的准确性和通过算法弥补缺漏的数据。最后生成“黄金”数据，用于情景计算。

\subsection{VaR系统设计}

本文采用的是历史模拟法进行 $\mathrm{VaR}$ 的计算, 无论选择何种模型, 都有存在着模型的风 险，模型风险定义为由于不恰当的参数、数据或者不恰当的风险度量模型造成损失的风险， VAR基础架构如图4所示。

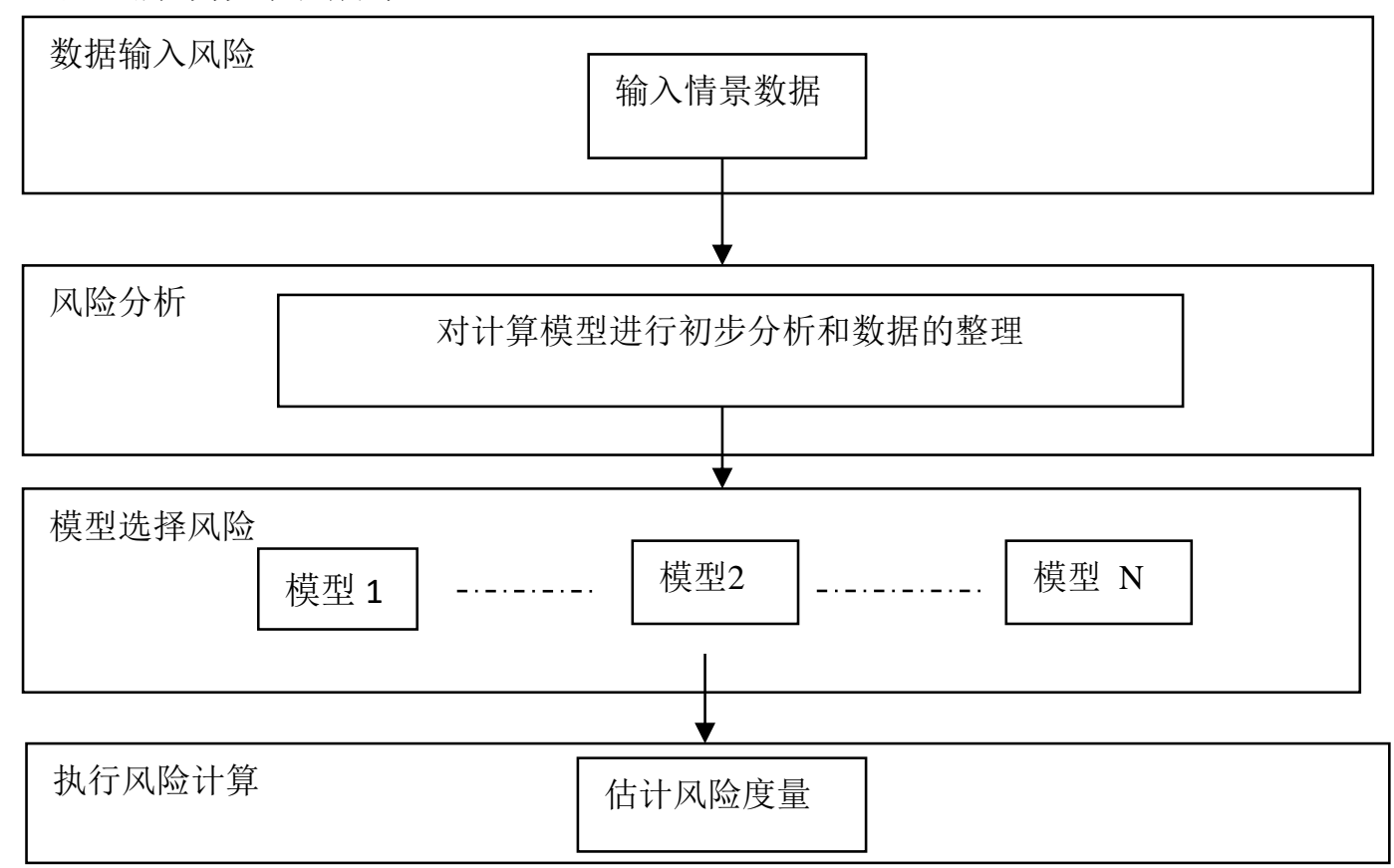

图4 VAR系统架构图 


\section{4. 实证分析}

风险价值系统的情景分析系统，可以计算包括外汇，股票，利率和信用风险。以股票的 情景分析为例进行实例探讨, 本案例数据来源于向彭博社, MarkIT购买和企业的内部数据。 根据所设计的系统, 可以相应的获得原始数据到, 最终转化为报酬率的过程。

(1) 原始数据

以谷歌股票价格的数据为例, 从2014年3月27到2015年9月27日的原始数据, 对其进行标 准化和聚合化。

(2) 将过去彼此相邻日期股票价格相减, 就可以求得该资产每日的价格损益变化量, 如 下图中的Price Value; 该资产在未来一天损益的可能情况（共有 $\mathrm{N}$ 种可能情形），将变化量 转换成报酬率，就可以算出可能报酬率，如表1中的Rate

表 1 谷歌股票的报酬率

\begin{tabular}{|r|r|r|r|}
\hline \multicolumn{2}{|c|}{ Date Close } & Price Value & \multicolumn{2}{l|}{ Rate } \\
\hline 25-Sep-15 & 611.97 & -13.83 & -0.0226 \\
\hline $24-S e p-15$ & 625.8 & 3.44 & 0.005497 \\
\hline $23-S e p-15$ & 622.36 & -0.33 & -0.00053 \\
\hline $22-S e p-15$ & 622.69 & -12.75 & -0.02048 \\
\hline $21-S e p-15$ & 635.44 & 6.19 & 0.009741 \\
\hline $18-S e p-15$ & 629.25 & -13.65 & -0.02169 \\
\hline $17-S e p-15$ & 642.9 & 6.92 & 0.010764 \\
$16-S e p-15$ & 635.98 & 0.84 & 0.001321 \\
\hline $15-S e p-15$ & 635.14 & 11.9 & 0.018736 \\
14-Sep-15 & 623.24 & -2.53 & -0.00406 \\
11-Sep-15 & 625.77 & 4.42 & 0.007063 \\
10-Sep-15 & 621.35 & 8.63 & 0.013889
\end{tabular}

(3) 通过对Rate进行分类和统计，生成正态分布曲线，如图5所示:

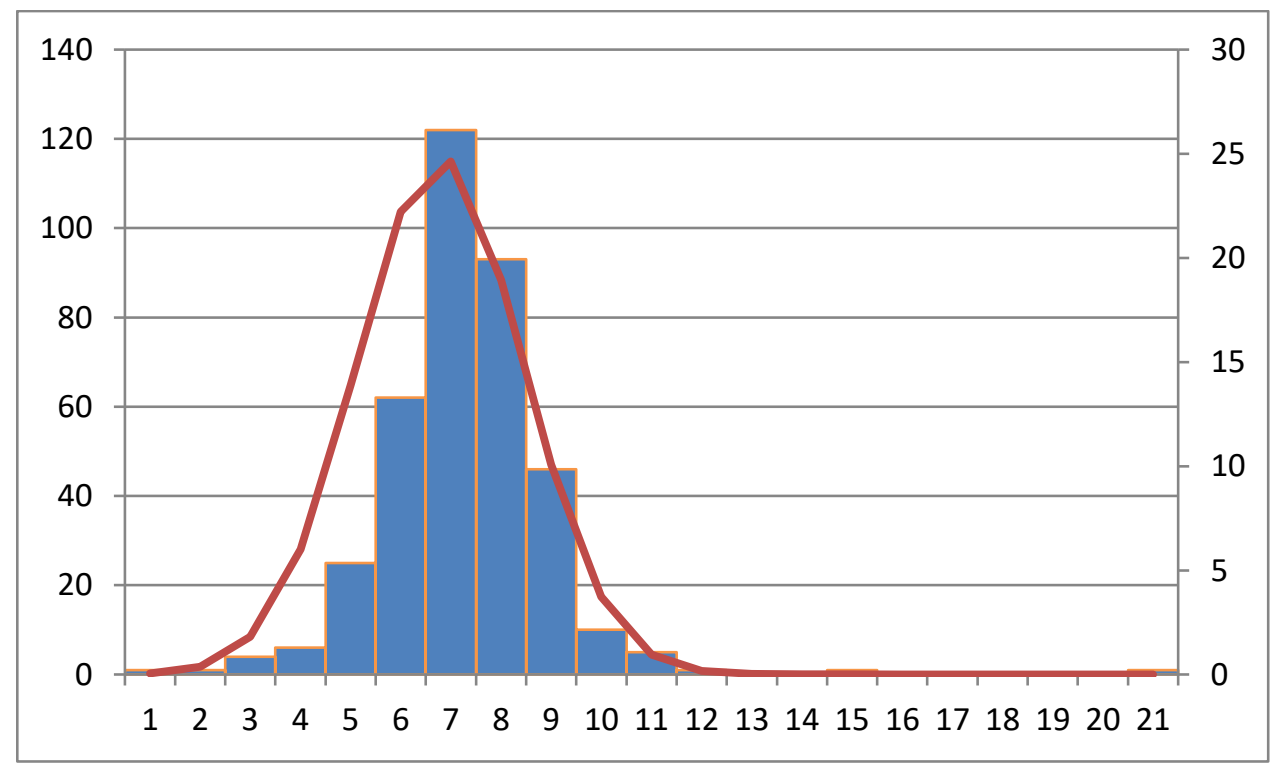

图5 VaR正态分布图

（4）根据置信区间，计算VaR的值，本文选用的置信区间是99\%，样本区间为2014-2015 年间, 379 个连续交易日的数据, $1 \%$ 的分位数是第 3 个, 假设投资股票为 1 百万美金, 那么 $\mathrm{VaR}=$ $100,0000 * 0.04287=42870$ 美元。也就是说持有该股票一天，损失额超过42870美元的可能性只 有 $1 \%$ 。根据以上方法也可以使用不同的情景数据进行输入, 计算在不同情况下的 $\mathrm{VaR}$ 值, 以 
此来监控银行每笔投资的风险敞口。由于银行每天要计算大量的 $\mathrm{VaR}$ 值, 因此优秀的架构对 于提高计算效率也是非常重要的。

\section{5. 结论}

本文通过讨论情景分析的方法, 对数据准确性进行讨论, 详细阐述了系统的实现。在实 证分析部分, 展现了谷歌近年的股票变化的 $\mathrm{VaR}$ 如何计算, 展现了一个VaR计算的过程, 通 过实例分析验证系统的可行性。学者对 $\mathrm{VaR}$ 的计算与研究主要是从计算模型的精确性进行研 究, 但VaR的计算是一个复杂的系统, 除了计算模型之外, 更应该以系统的观点来分析 VaR 计算, 因此本文对 $\mathrm{VaR}$ 前端的情景分析, 数据准确性进行了精确的论述, 对系统架构进行了 详细的论述。以系统的视角对商业银行情景计算系统和 VaR计算系统的架构进行描述, 对商 业银行实施情景分析，进行VaR系统建设有重要的辅助和启示作用。

\section{致谢}

本文为国家社科基金一般项目《大数据背景下金融市场信用违约预测和违约风险补偿研 究》(15BJY149)的阶段性成果之一。

\section{References}

[1] IMF, BIS, FSB. Guidance to assess the systemic importance of financial institutions, markets and instruments: initial considerations, Report to the G20 Finance Ministers and Governors, 2009.

[2] Adrian T, Brunnermeier M. CoVaR. Federal Reserve Bank of New York, Staff Reports, No.348, 2008.

[3] Kaminsky G. S. Lizondo M. Reinhart. Leading indicators of currency crises. IMF Working paper, 45(1):1-48, 1998.

[4] Sachs J, Tornell A, Velasco A. The Mexican peso crises: sudden death or death foretold, Journal of International Economics, Vol. 41, pp: 265-283, 1996.

[5] Frankel, Jeffrey A. Rose. Currency crashes in emerging markets: an empirical treatment. Journal of International Economics, Vol.41, pp:351-368, 1996.

[6] Lehar A. Measuring systemic risk: a risk management approach. Journal of Banking and Finance, Vol.29, pp:20-30, 2005.

[7] Ma Junlu, Fan Xiaoyun, Cao Yuantao, Estimating Bilateral Exposures in the China

Interbank Market :Is There a Systemic Contagion? Economic Research Journal, Vol.1, pp:68-78,2007.

[8] Muller J. Interbank credit lines as a channel of contagion, Journal of Financial Services Research, Vol.29, pp:37-60, 2006.

[9] Schroder M, Schuler M. The systemic risk in European banking-evidence from bivariate GARCH models, Centre for European Economic Research, Working Paper, 2003.

[10] Huang, Hao Zhou, and Haibin Zhu. A framework for assessing the systemic risk of major financial institution, Journal of Banking and Finance, Vol.33, pp:2036-2049, 2009. 
[11] Tarashev, Nikola, C. Borio and K. Tsatsaronis. Attributing systemic risk to individual institutions: methodology and policy implications, BIS Working Paper, No 308, 2010.

[12] Drehmann M and N Tarashev. Measuring the systemic importance of interconnected banks, BIS Working Paper, No.342, 2011.

[13] Balakrishnan, R. et al. How financial stress spreads: a first comprehensive look at the current crisis. 2009, http//: www.voxeu.org /index.php $q=$ node/3500.

[14] Adams, Z., R. Füss and R. Gropp. Modeling spillover effects among financial institutions: a state-dependent sensitivity Value-at-Risk ( SDSVaR ) approach, EBS Working Paper, 2010.

[15] Wong, A., and T. Fong. An analysis of the interconnectivity among the Asia-Pacific economies, Hong Kong Monetary Authority, Working Paper, 2010.

[16] Gauthier, C., A. Lehar, and M. Souissi. Macro prudential capital requirements and systemic risk, Bank of Canada, Working Paper, 2009.

[17] YE Wu-yi, MIAO Bai-qi, Evaluation of conditional VaR based on threshold quantile regression model, Journal of Systems Engineering, Vol.2, pp:154-160, 2008.

[18] YE Wu-yi, CHEN Jie-cheng, MIAO Bai-qi, Estimating of Conditional VaR and Analysis of Leverage Effect Based on Dull Variable Quantile Regression Model, Chinese Journal of Management Science, Vol.4, pp:1-7, 2010.

[19] Xie Fuzuo, Research on Risk Spillover Effect Based on CoVaR Measure, Journal of Financial Development Research, Vol.6, pp:59-63, 2010.

[20] Jing Mao, Meng Luo, Research on Spillover Effect between Banking and Securities Industry -- Based on CoVaR Model, New Finance, Vol.267, pp:21-27, 2011.

[21] Zhang Qiang, Feng Cha, The Measurement of Systemic Risk of China's Listed Commercial Banks after Financial Crisis, Shanghai Finance, Vol.12, pp:32-35, 2010.

[22] LI Zhi-hui, FAN Li, Empirical Study on Systemic Risk Spillover of China Commercial Banks, Modern Economic Science, Vol.11, pp:13-20, 2011.

[23] GAO Guo-hua, PAN Ying-li, Banking Systemic Risk Based on Dynamic CoVaR Estimation, Journal of Shanghai Jiaotong University, Vol.45(12), pp:1753-1759, 2011.

[24] Zhou Tianyun, Zhou Kaiguo, Huang Lian, Institution Agglomeration, Risk Contagion and Systemic Risk of Hongkong bank, Studies of International Finance, Vol.4, pp:77-87, 2012.

[25] FSB. Policy Measures to address systemically important financial institutions, Financial Stability Board, Vol.38,2011. 